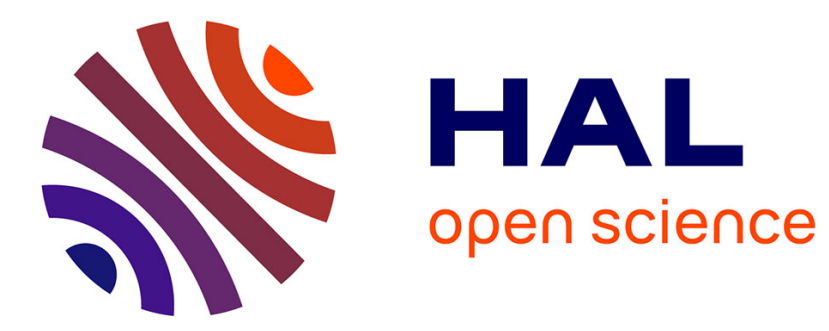

\title{
Compact infrared cryogenic wafer-level camera: design and experimental validation
}

\author{
Florence de La Barrière, Guillaume Druart, Nicolas Guérineau, Gilles \\ Lasfargues, Manuel Fendler, Nicolas Lhermet, Jean Albert Taboury
}

\section{- To cite this version:}

Florence de La Barrière, Guillaume Druart, Nicolas Guérineau, Gilles Lasfargues, Manuel Fendler, et al.. Compact infrared cryogenic wafer-level camera: design and experimental validation. Applied optics, 2012, 51 (8), pp.1049-1060. hal-00701492

\section{HAL Id: hal-00701492 \\ https://hal-iogs.archives-ouvertes.fr/hal-00701492}

Submitted on 25 May 2012

HAL is a multi-disciplinary open access archive for the deposit and dissemination of scientific research documents, whether they are published or not. The documents may come from teaching and research institutions in France or abroad, or from public or private research centers.
L'archive ouverte pluridisciplinaire HAL, est destinée au dépôt et à la diffusion de documents scientifiques de niveau recherche, publiés ou non, émanant des établissements d'enseignement et de recherche français ou étrangers, des laboratoires publics ou privés. 


\title{
Compact infrared cryogenic wafer-level camera: design and experimental validation
}

\author{
Florence de la Barrière, ${ }^{1, *}$ Guillaume Druart, ${ }^{1}$ Nicolas Guérineau, ${ }^{1}$ Gilles Lasfargues, ${ }^{2}$ \\ Manuel Fendler, ${ }^{2}$ Nicolas Lhermet, ${ }^{2}$ and Jean Taboury ${ }^{3}$ \\ 'ONERA, The French Aerospace Lab, Chemin de la Hunière, 91761 Palaiseau cedex, France \\ ${ }^{2}$ CEA LETI MINATEC, 17 rue des Martyrs, 38054 Grenoble cedex, France \\ ${ }^{3}$ Laboratoire Charles Fabry, Institut d'Optique, CNRS, Univ Paris Sud, 2 avenue Augustin Fresnel, \\ 91127 Palaiseau cedex, France \\ *Corresponding author: florence.de_la_barriere@onera.fr
}

Received 23 November 2011; accepted 9 January 2012;

posted 12 January 2012 (Doc. ID 158765); published 5 March 2012

\begin{abstract}
We present a compact infrared cryogenic multichannel camera with a wide field of view equal to $120^{\circ}$. By merging the optics with the detector, the concept is compatible with both cryogenic constraints and waferlevel fabrication. The design strategy of such a camera is described, as well as its fabrication and integration process. Its characterization has been carried out in terms of the modulation transfer function and the noise equivalent temperature difference (NETD). The optical system is limited by the diffraction. By cooling the optics, we achieve a very low NETD equal to $15 \mathrm{mK}$ compared with traditional infrared cameras. A postprocessing algorithm that aims at reconstructing a well-sampled image from the set of undersampled raw subimages produced by the camera is proposed and validated on experimental images. (C) 2012 Optical Society of America

OCIS codes: $\quad 110.3080,220.4830,110.4190,110.4155,350.3950,080.2730$.
\end{abstract}

\section{Introduction}

Nowadays, both civilian and military applications require miniaturized and cheap optical systems. The constraints on the size and weight of such systems are so demanding that downscaled versions of traditional systems with a single optical axis are reaching their limits because of a loss of resolved points in the final image [1]. A solution to compensate for this problem can be found by looking at nature, where small invertebrates have developed compound eyes [2]. Therefore, mutiaperture imaging seems to be a good approach to designing thin optical systems. Several multichannel systems inspired by the vision of invertebrates have already been proposed [3-15]. All these concepts tend to merge the optical system

$1559-128 \mathrm{X} / 12 / 081049-12 \$ 15.00 / 0$

(C) 2012 Optical Society of America with the detector, leading to very thin wafer-level camera modules.

Two main multichannel approaches, which differ in the way of dividing the information contained in the scene, can be distinguished. On the one hand, each channel captures a low-resolution image of the overall field of view (FOV), which is known as the thin observation module by bound optics (TOMBO) principle $[3,14,15]$. Providing nonredundant information between the subimages [16], a high resolution image is retrieved thanks to a superresolution image processing $[3,17-21]$. On the other hand, the spatial content of the scene can be distributed between the several channels $[4-6, \underline{6}, 10-12]$. Each channel has a reduced FOV, and the optical axes of the different channels are tilted with respect to each other so that each channel images a small part of the scene. Then, an image processing method has to be applied to stitch all the images together and to reconstruct a final image. 
Most of these concepts operate in the visible spectral range [3-7,9-11]. Few address the infrared spectral range: the systems of $[14,15]$ work in the

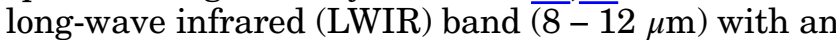
uncooled microbolometer detector array, and the concept of [12] addresses a narrow spectral bandwidth around $\overline{1064} \mathrm{~nm}$.

Our field of research addresses cooled infrared detectors operating in the midwave infrared (MWIR) band $(3-5 \mu \mathrm{m})$. The infrared focal plane arrays (IRFPAs) we traditionally use are sensitive quantum detectors based on $\mathrm{HgCdTe}$ technology. They need to be cooled at liquid nitrogen temperature $(77 \mathrm{~K})$, which is why they are integrated in a compact Dewar illustrated in Fig. 1. A Dewar is a vacuum chamber closed by a window; a cold shield and a cold diaphragm limit the angle of view of the detector, in order to avoid background emission from the external roof of the Dewar. Because of these packaging conditions, an infrared cryogenic camera has to fulfill several requirements: its optical architecture has to be as simple as possible, and it must have a minimal size. Our approach first consists in integrating a very simple system in the cold shield: it only relies on a traditional lens that images the scene with a very good optical quality [22]. We have also proposed in previous work [8] a cooled multichannel optical system integrated in the cold shield with tilted optical axes to increase the overall FOV. The next step consists in going further in the miniaturization process by integrating an extremely compact optical system directly on the IRFPA, leading to a cooled wafer-level camera module.

This paper aims at demonstrating that a very compact and a large FOV multichannel system can be designed by applying the TOMBO principle in the field of cooled MWIR IRFPAs. Our solution leads to a thin wafer-level cryogenic infrared camera, which is completely adapted to the demanding packaging environment of IRFPAs operating between $3 \mu \mathrm{m}$ and $5 \mu \mathrm{m}$.

Section 2 describes the design strategy of a compact multichannel camera, and Section 3 depicts how technological constraints impact this design.
Section 4 gives an insight into the starting point and the optimization of the optical design. Section 5 is an overview of the technological realization of the component. Section 6 deals with the experimental characterization of this camera: we have measured its modulation transfer function (MTF), as well as its noise equivalent temperature difference (NETD). We have also developed a postprocessing algorithm that combines the diversity of information contained in the raw subimages produced by the camera to reconstruct a single well-sampled image.

\section{Design Strategy Leading to a Compact Multichannel Camera}

\section{A. Decreasing the Focal Length: Large FOV System}

In previous work [23], we have discussed several strategies to miniaturize optical systems. One of these consists in decreasing the focal length $f$ of the optical system, which is known as the TOMBO principle [3]. By decreasing $f$, the amount of aberrations decreases, and thus a simple system based on a single lens only can easily be limited by the diffraction. Under paraxial conditions, the focal length $f$ is linked to the FOV of the system with the following equation:

$$
f=\frac{t_{\mathrm{det}}}{2 \tan (\mathrm{FOV} / 2)}
$$

where $t_{\text {det }}$ is the size of the detector. Therefore, choosing a very small focal length $f$ for the optical system results in a wide FOV.

However, the main problem that arises when decreasing the focal length is the loss of angular resolution of the optical system. In the next subsections, we define the angular resolution, and we discuss how this limitation can be overcome.

\section{B. Angular Resolution of an Optical System}

The maximum resolvable spatial frequency $\nu_{\max }$ of an optical system is the ability to distinguish small details, which we define by the following equation:

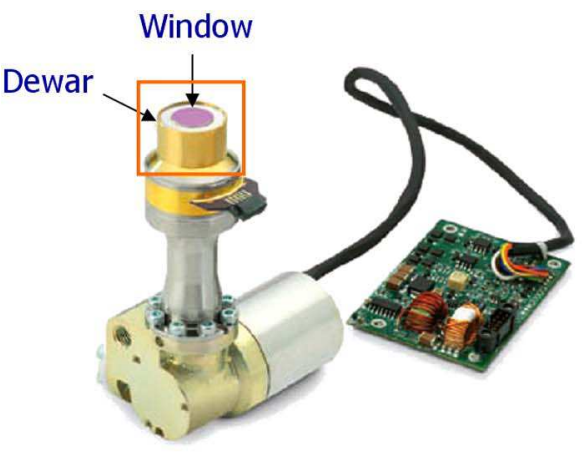

(a)

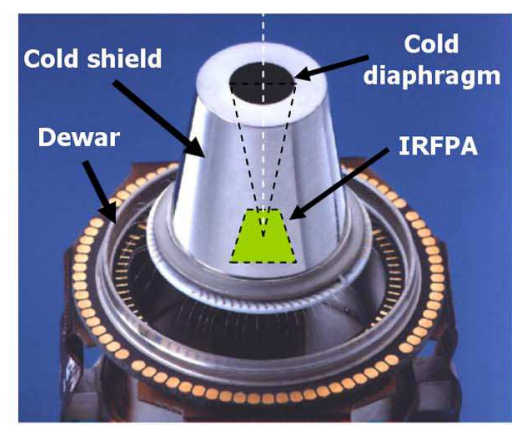

(b)

Fig. 1. (Color online) (a) External view of a Dewar commercialized by the French company Sofradir. (b) Internal view of a Dewar (corresponding to the orange frame represented in (a)). 


$$
\nu_{\max }=\min \left(1 /\left(2 p_{s}\right), 1 / t_{\mathrm{pix}}, \nu_{c}\right),
$$

where $p_{s}$ is the sampling pitch of the focal plane array (FPA), $t_{\text {pix }}$ is the size of the active zone of the pixel, and $\nu_{c}$ is the cutoff frequency of the optical system.

From this equation, it turns out that the resolution of an optical system can be affected by three main contributors, which are illustrated in Fig. 2: the optical cutoff frequency $\nu_{c}$, the Nyquist frequency $1 /\left(2 p_{s}\right)$ associated with the FPA, and the pixel cutoff frequency $1 / t_{\text {pix. }} 1 / t_{\text {pix }}$ (respectively $\nu_{c}$ ) is the spatial frequency for which the modulus of the pixel transfer function (respectively the optical transfer function) is equal to 0 .

For most traditional optical systems, the angular resolution is limited by the Nyquist frequency associated with the FPA. If we want $\nu_{\max }$ to be limited by the cutoff frequency of the pixel (assuming that the quality of the optical system is good enough to provide an optical cutoff frequency greater than $\left.1 / t_{\text {pix }}\right)$, we have to propose innovative multichannel designs.

\section{Designing a Multichannel Optical System to Artificially Increase the Nyquist Frequency}

In previous work [24], we have shown that a system based on the TOMBO principle (i.e., a multichannel system that provides subpixel shifts between the subimages) associated with a FPA of sampling pitch $p_{s}$ is equivalent to sampling one subimage with a monodetector of size $p_{s}$, which is scanned with a pitch equal to $p_{s} / 2$ in the two directions (if parallax effects are not taken into account). In this case, the Nyquist frequency is artificially increased to the value $1 / p_{s}$ so that no aliasing effects occur anymore. Moreover, we can notice that this artificial Nyquist frequency $1 / p_{s}$ is also the cutoff frequency of a pixel of size $p_{s}$ (which is the maximum frequency we can expect to retrieve). Then, the resolution of the system becomes limited by the pixel cutoff frequency. To increase the range of accessible spatial frequencies, the size of the pixel can be decreased until the technological limit is reached. If we want to go beyond the pixel cutoff frequency, we can decrease the fill factor of the pixels as explained in the next subsection.

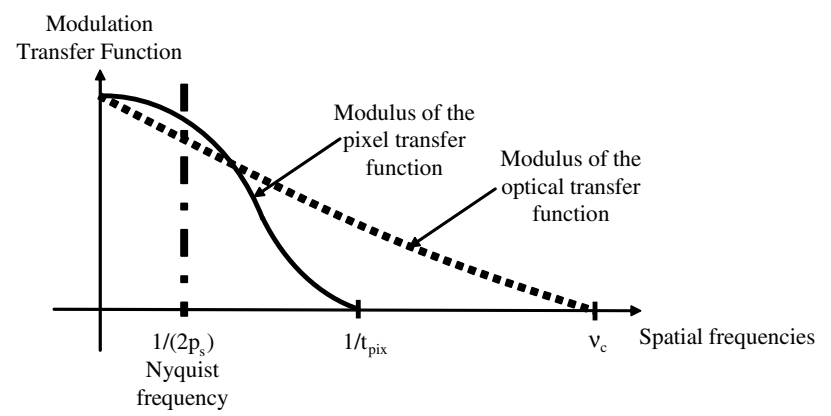

Fig. 2. Illustration of the three contributors to the angular resolution of an optical system: the Nyquist frequency $1 /\left(2 p_{s}\right)$, the pixel cutoff frequency $1 / t_{\mathrm{pix}}$, and the optical cutoff frequency $\nu_{c}$.
D. Decreasing the Fill Factor of the Pixels to Increase the Pixel Cutoff Frequency

If we want to retrieve higher spatial frequencies, we have to decrease the size of the pixel $t_{\text {pix }}$ while keeping a sampling pitch $p_{s}$ constant, because the sampling pitch cannot undergo further reduction. This is equivalent to decreasing the fill factor of the pixels, which is defined by the following equation:

$$
F=\left(\frac{t_{\text {pix }}}{p_{s}}\right)^{2} .
$$

The optimal number of nonredundant channels that is needed to provide nonredundant information is equal to [23]

$$
N_{\mathrm{ch}}=\frac{4}{F} \text {. }
$$

Thus, the design strategy can be summarized in two main steps:

1. Designing a multichannel system that provides nonredundant information in the subimages enables us to increase the Nyquist frequency to the value of the pixel cutoff frequency, in order to not be limited by the Nyquist frequency.

2. The fill factor of the pixels decreases so that the accessible range of spatial frequencies is widened.

In this design strategy, we assume that the quality of the optical system is good enough so that the optical cutoff frequency remains higher than the pixel cutoff frequency. Then, the resolution of the system is limited by the pixel cutoff frequency.

E. Increase of the Number of Resolved Points in the Final Image Produced by a Multichannel Camera

The number of resolved points $N_{b}$ is defined as follows [23]:

$$
N_{b}=\left(\frac{\mathrm{FOV}}{\mathrm{IFOV}}\right)^{2}
$$

where the instantaneous field of view (IFOV) is given by

$$
\mathrm{IFOV}=\frac{1}{f \nu_{\max }} .
$$

The angular resolution of a single channel is limited by the Nyquist frequency, so that $\mathrm{IFOV}_{\mathrm{ch}}=$ $2 p_{s} / f$, whereas the angular resolution of the multichannel system combined with a postprocessing algorithm is limited by the cutoff frequency of the pixel, so that IFOV multich $=t_{\text {pix }} / f$.

Then, if we compare the number of resolved points of the multichannel system $N_{b}$ multich with the number of resolved points of a single channel $N_{b}{ }^{\text {ch }}$, we 
find that the theoretical increase in the number of resolved points is equal to the number of optical channels:

$$
N_{b} \text { multich }=\frac{4}{F} N_{b} \text { ch }
$$

\section{Impact of Technological Constraints on Optical Design}

An important point when designing a wafer-level camera based on the principles described in Section 2 is to take into account the technological constraints linked to the manufacturing of the optical module and its assembly near the IRFPA.

\section{A. Manufacturing Small Focal Length Microlens Arrays}

As explained in Section 2, the focal length of each channel needs to be small, which results in an important sag height for the microlenses. Two approaches can be carried out to manufacture microlens arrays. On the one hand, diamond turning techniques enable obtaining very important sag heights [25]; however, each microlens is manufactured separately, and thus, diamond turning is not compatible with wafer-level fabrication. On the other hand, standard semiconductor technologies involving photolithography, resist processing, and reactive ion etching are well suited for wafer-level fabrication [26]; however, sag heights equal to or greater than approximately $100 \mu \mathrm{m}$ are difficult to obtain. Therefore, while designing the optical system, we have to keep in mind that the maximum sag height is limited to $100 \mu \mathrm{m}$. Moreover, plano-convex microlenses are easily manufactured at wafer-level without requiring any precise alignment procedure between the two sides of the wafer. Therefore, in the optical design, we choose to use microlenses with one curved surface only.

\section{B. Assembling the Optical Module Near the IRFPA}

The optical wafer-level module is assembled with the IRFPA using advanced, innovative, and precise techniques developed at CEA LETI MINATEC. These techniques are directly inspired by the hybridization of an IRFPA. Basically, an IRFPA based on $\mathrm{HgCdTe}$ technology is made of an HgCdTe layer (which is sensitive to infrared radiations) and of a silicon readout circuit. The HgCdTe layer and the Silicon readout circuit are connected with indium bumps. The idea is to support the optical module at a short distance from the HgCdTe layer with indium bumps also. The whole assembling process is compatible with wafer-level fabrication and is adapted to the cryogenic environment. This kind of integration, which relies on state-of-the-art technology developed at CEA LETI MINATEC, implies that the back focal length (BFL), which is defined as the distance between the last diopter and the detector, remains inferior to approximately $700 \mu \mathrm{m}$.

The optical design may require one or several diaphragm layers to provide the pupil of the system and to solve the problem of crosstalk between adjacent channels (a description of the issues linked to crosstalk in the specific case of our system will be discussed in Subsection 4.C). The diaphragm layers can be made either of independent metallic arrays of pinholes or of a metallization on the surfaces of the lenses. From a practical point of view, an independent metallic pinhole array would be supported using the same hybridization techniques as the microlenses. However, in order to avoid stacking too many elements, we prefer using a metallization on the surfaces of the lenses.

The technological constraints and their impact on optical design are summarized in Table $\underline{1}$.

\section{Optical Design: Starting Point and Optimization}

In the two next subsections, we focus on the design of one optical channel while keeping in mind that it has to be replicated to obtain a multichannel system. The idea is to find which simple optical architecture is best suited to be integrated at a short distance from the IRFPA.

\section{A. Simple Optical System Based on a Single Lens}

The simplest system is composed of a diaphragm, a single optical component, and a detector [23]. The optical component is a plano-convex lens, for which the curved surface can be turned towards the detector [cf. Fig. 3(a)] or towards the scene [cf. Fig. 3(b)]. We have studied the BFL for these simple optical systems under paraxial conditions using ray-transfer matrix formalism, which aims at identifying the system that has the smallest BFL for a given focal length $f$.

The ray-transfer matrix formalism is commonly used for a paraxial description of thick optical systems [27]. The entire optical system (from the entrance pupil to the detector) is represented by a matrix $M$ that is derived from the multiplication of all the individual element matrices of the lenses and the corresponding propagation between them. The ray height $h_{\text {out }}$ and the angle $\alpha_{\text {out }}$ to the optical axis of the output ray are calculated from the ray height $h_{\text {in }}$ and angle $\alpha_{\text {in }}$ of the incident ray by

$$
\left(\begin{array}{c}
h_{\text {out }} \\
\alpha_{\text {out }}
\end{array}\right)=M \cdot\left(\begin{array}{c}
h_{\text {in }} \\
\alpha_{\text {in }}
\end{array}\right) .
$$

Table 1. Impact of Technological Constraints on the Optical Design of an Infrared Cryogenic Wafer-Level Camera

\begin{tabular}{ll}
$\begin{array}{c}\text { Technological } \\
\text { constraint }\end{array}$ & \multicolumn{1}{c}{$\begin{array}{c}\text { Solution compatible with } \\
\text { state-of-the-art technology }\end{array}$} \\
\hline Simple system & $\begin{array}{c}\text { Minimal number of optical elements } \\
\text { (a diaphragm, a detector, and one or } \\
\text { two lenses) } \\
\text { Plano-convex microlens arrays } \\
\text { Type of lenses }\end{array}$ \\
Diaphragms & $\begin{array}{l}\text { Metallization on the surfaces of the } \\
\text { microlens arrays }\end{array}$ \\
Sag height & $\begin{array}{l}\text { Inferior to approximately } 100 \mu \mathrm{m} \\
\text { BFL }\end{array}$
\end{tabular}




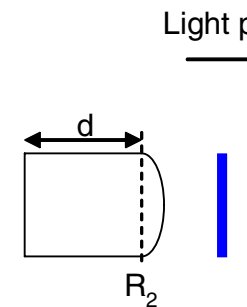

(a)

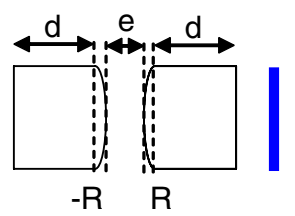

(c)

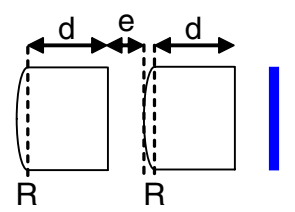

(e)

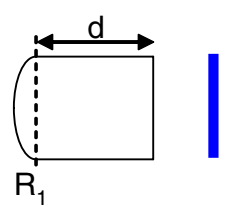

(b)

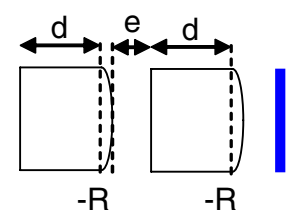

(d)

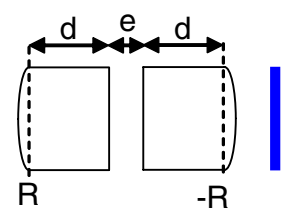

(f)
Fig. 3. (Color online) Schematic layouts of six simple optical systems and notations. (a) Plano-convex lens with the curved face towards the detector (thickness $d$ and radius of curvature $R_{2}$, $R_{2}<0$ ). (b) Plano-convex lens with the curved face towards the scene (thickness $d$ and radius of curvature $R_{1}, R_{1}>0$ ). (c), (d), (e), and (f) Four possible associations of two plano-convex lenses with the same radius of curvature $(R>0)$ and with thickness $d$. Light propagates from the left to the right; the blue line at the right stands for the detector.

The BFL of the plano-convex lenses illustrated in Figs. 3(a) and 3(b) can be derived from the general case of a bi-convex lens with radii of curvature $R_{1}$ and $R_{2}\left(R_{1}>0\right.$ and $\left.R_{2}<0\right)$ [cf. Fig. 4]. The detailed calculation of $f$ and BFL using ray-transfer matrix formalism for a bi-convex lens is recalled in Appendix A. The expressions of the focal length $f$ and of the BFL of the systems illustrated in Figs. 3(a) and 3(b) (for which $R_{1}=\infty$ and $R_{2}=\infty$, respectively) are derived from Eqs. (A7) and (A8), and they are given in Table $\underline{2}$.

A plano-convex lens with the curved surface towards the scene exhibits a BFL smaller than $f$, whereas a plano-convex lens with the curved surface

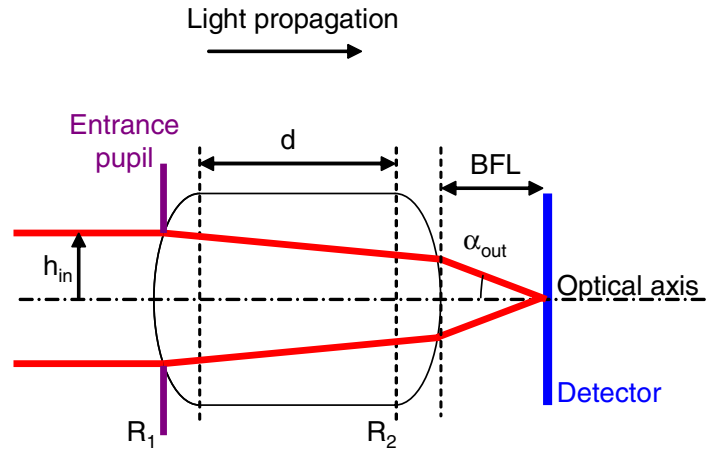

Fig. 4. (Color online) Optical layout of a bi-convex lens. The notations for the calculation of BFL using ray-transfer matrix formalism are provided.

towards the detector has a BFL equal to $f$. Therefore, the system of Fig. 3(b) seems to be well suited to reduce the BFL. However, the predominant aberration of an infrared system is astigmatism, which can be corrected by moving the pupil away from the surface that carries optical power [28]. Therefore, this solution is not efficient if we want to make a metallization on the entrance curved surface.

Then, we choose the plano-convex lens with the curved face towards the detector as the starting point for an optical design based on a single lens. The FOV is equal to $120^{\circ}$. We choose to use an IRFPA with a reduced fill factor equal to $F=0.25$. The size of the active zones will be equal to $t_{\text {pix }}=15 \mu \mathrm{m}$, and they will be implanted in pixels of size $p_{s}=30 \mu \mathrm{m}$. Then, the design turns to a $4 \times 4$ multichannel system [cf. Eq. (4)]. The nonredundancy between the subimages is obtained by choosing a period for the microlenses that is not a multiple of the detector sampling pitch $p_{s}$. We provide a shift equal to $7.5 \mu \mathrm{m}$ between adjacent channels in the two directions; then the microlens array's pitch is theoretically equal to $1.8975 \mathrm{~mm}$ in the two directions. Therefore, each channel projects an image onto an area of about $64 \times 64$ pixels. As the format of the IRFPA is equal to $320 \times 256$ pixels, we use all the pixels of the detector in the direction of the 256 pixels. The optical quality of one channel was optimized using the commercial software Zemax, and its layout is shown in Fig. 5. It is made of a single plano-convex silicon microlens array. Table $\underline{3}$ sums up the optical characteristics of the multichannel system.

Table 2. Focal Length and BFL of the Simple Optical Systems Illustrated in Fig. 3

\begin{tabular}{cll}
\hline System & Focal length $f$ & BFL \\
\hline (a) & $f=\frac{-R_{2}}{n-1}$ & BFL $=f$ \\
(b) & $f=\frac{R_{1}}{n-1}$ & BFL $=f\left(1-\frac{d}{R_{1}} \frac{n-1}{n}\right)$ \\
(c) & $\frac{1}{f}=2 \frac{n-1}{R}-e \frac{(n-1)^{2}}{R^{2}}$ & BFL $=f\left(1-2 \frac{d}{R} \frac{n-1}{n}-e \frac{n-1}{R}-\frac{d e}{R^{2}} \frac{(n-1)^{2}}{n}\right)$ \\
(d) & $\frac{1}{f}=2 \frac{n-1}{R}-\left(e+\frac{d}{n}\right) \frac{(n-1)^{2}}{R^{2}}$ & BFL $=f\left[1-\frac{n-1}{R}\left(e+\frac{d}{n}\right)\right]$ \\
(e) & $\frac{1}{f}=2 \frac{n-1}{R}-\left(e+\frac{d}{n}\right) \frac{(n-1)^{2}}{R^{2}}$ & BFL $=f\left[1-\left(e+\frac{3 d}{n}\right) \frac{n-1}{R}+\frac{(n-1)^{2}}{n}\left(e+\frac{d}{n}\right) \frac{d}{R^{2}}\right]$ \\
(f) & $\frac{1}{f}=2 \frac{n-1}{R}-\left(e+\frac{2 d}{n}\right) \frac{(n-1)^{2}}{R^{2}}$ & BFL $=f\left[1-\frac{n-1}{R}\left(e+\frac{2 d}{n}\right)\right]$ \\
\hline
\end{tabular}




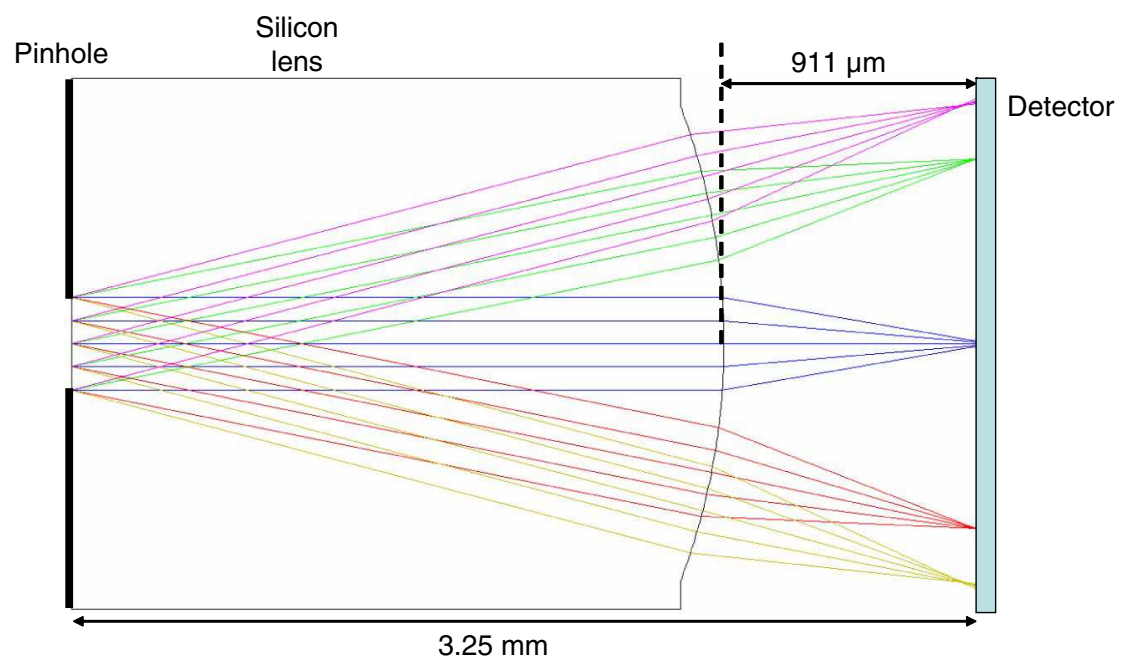

Fig. 5. (Color online) Optical layout of one channel of an infrared wafer-level camera based on a single plano-convex lens. The different colors stand for different field angles.

Despite the optical simplicity of this solution, manufacturing the microlens array is difficult and not compatible with wafer-level fabrication, since the lens sag height is greater than $100 \mu \mathrm{m}$. Moreover, the thickness of the microlens on the optical axis $(2.3 \mathrm{~mm})$ is too high for current state-of-the-art technology, and the BFL is not compatible with hybridization techniques.

Then we need to use two stages of microlenses in the optical design. In this way, the optical power may be divided into two diopters, each one having a reduced sag height.

\section{B. Simple Optical System Based on Two Lenses}

We study systems based on only two plano-convex microlenses. For manufacturing purposes, it is easier to consider the case in which the two lenses have the same radius of curvature. Four possible associations (illustrated in Figs. 3(c), 3(d), 3(e), and 3(f)) can be studied in terms of $\mathrm{BFL}$ and $f$. The results are gathered in Table $\underline{2}$.
For the systems illustrated in Figs. 3(c), 3(d), and $3(\mathrm{f}), \mathrm{BFL}$ is smaller than $f$. However, the expression of BFL for system (e) is not easily interpretable. But it is worth mentioning that, for systems (e) and (f), astigmatism could not be corrected efficiently if the diaphragm was made of a metallization on the first curved surface of the assembly.

Therefore, we have to choose between systems (c) and (d). The optical quality of one channel was optimized for these two systems using the commercial software Zemax, and the best optical quality was obtained for system (c). Its layout is shown in Fig. 6. It is made of two plano-convex silicon microlenses with the curved face in front of each other. Table 3 sums up the optical characteristics of the multichannel system.

\section{Diaphragms and Prevention from Crosstalk}

To prevent the multichannel system from crosstalk, we provide three layers of diaphragms, which rely on the surface available between adjacent microlenses.

Table 3. Optical Characteristics of the Multichannel Architectures Corresponding to Fig. 5 (One Lens) and Fig. 6 (Two Lenses)

\begin{tabular}{|c|c|c|}
\hline Property & Value (one lens) & Value (two lenses) \\
\hline Size of optics module $(L \times W \times T)$ & $10 \mathrm{~mm} \times 10 \mathrm{~mm} \times 3.25 \mathrm{~mm}$ & $10 \mathrm{~mm} \times 10 \mathrm{~mm} \times 4.08 \mathrm{~mm}$ \\
\hline Field of view & $120^{\circ}$ & $120^{\circ}$ \\
\hline$f$-number of one channel & 3 & 3 \\
\hline Focal length of one channel & $993 \mu \mathrm{m}$ & $1.003 \mathrm{~mm}$ \\
\hline BFL of one channel & $911 \mu \mathrm{m}$ & $384 \mu \mathrm{m}$ \\
\hline Number of channels & $4 \times 4$ & $4 \times 4$ \\
\hline Thickness of microlenses on the optical axis & $2.3 \mathrm{~mm}$ & $1.8 \mathrm{~mm}$ \\
\hline Number of pixels per channel & $64 \times 64$ & $64 \times 64$ \\
\hline Microlens array pitch & $1.8975 \mathrm{~mm}$ & $1.8975 \mathrm{~mm}$ \\
\hline Diameter of microlenses & $1.7 \mathrm{~mm}$ & $1.7 \mathrm{~mm}$ \\
\hline Radius of curvature & $2.390 \mathrm{~mm}$ & $4.707 \mathrm{~mm}$ \\
\hline Microlenses height sag & $156 \mu \mathrm{m}$ & $77 \mu \mathrm{m}$ \\
\hline Pinhole diameter & $331 \mu \mathrm{m}$ & $335 \mu \mathrm{m}$ \\
\hline Optical quality & Limited by the diffraction & Limited by the diffraction \\
\hline
\end{tabular}




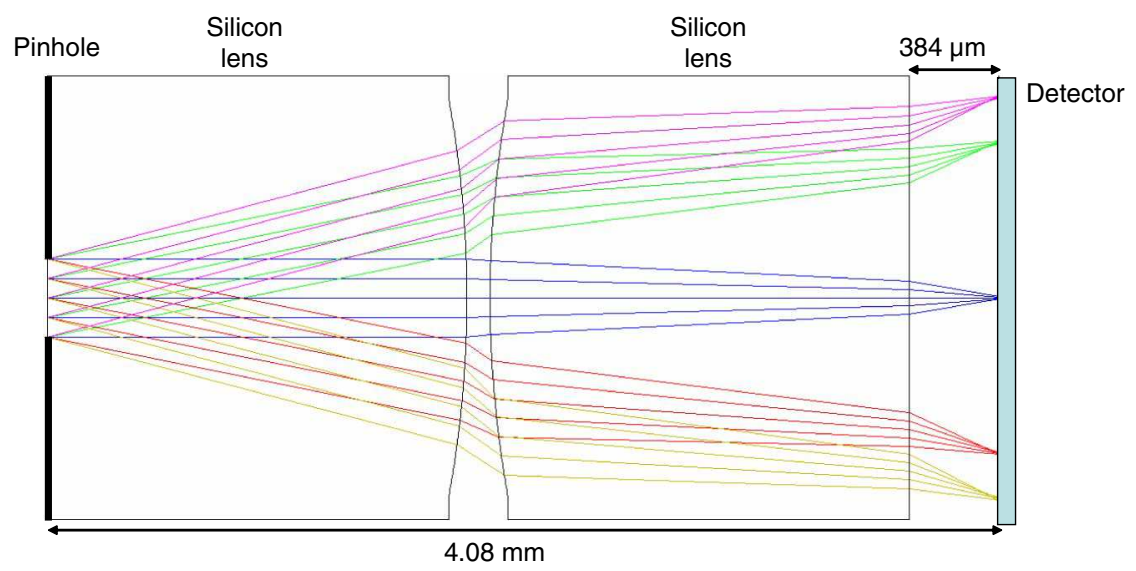

Fig. 6. (Color online) Optical layout of one channel of an infrared wafer-level camera based on two plano-convex lenses with the same radius of curvature. The different colors stand for different field angles.

A schematic view of the overall system with the layers of diaphragms is given in Fig. 7 .

The cold shield is also used to limit the angle of view of the system to a value equal to $120^{\circ}$. The ray corresponding to the maximum field angle for the first channel relies on the edge of the cold shield (cf. Fig. 7). Rays with an angle of incidence greater than $120^{\circ}$ also enter the cold shield and are within the FOV of other channels. The field diaphragm (which corresponds to the metallization on the flat face in front of the detector) blocks these rays, and we provide five "nonuseful" pixels between adjacent channels to account for the projection of the field diaphragm on the detector.

\section{Technological Realization of the Infrared Multichannel Wafer-Level Camera}

The microlens arrays were fabricated at a wafer-level using traditional techniques [26], which enable a very accurate shaping of the lens profile and a precise positioning of the lenses within an array. The manufacturing (lens profile, antireflective coating, chromium and gold diaphragm arrays metallization) has been made by the company SUSS MicroOptics using $200 \mathrm{~mm}$ wafer technology.
An IRFPA based on HgCdTe technology with a small pixel fill factor has been made specifically for this wafer-level camera by CEA LETI MINATEC. Then, the optical wafer-level module was assembled with the detector using innovative and precise techniques that are directly inspired by the hybridization of an IRFPA. The whole system is illustrated in Fig. 8 . It has been integrated in a Dewar and characterize $\bar{d}$.

\section{Characterization of the Cryogenic Wafer-Level Infrared Camera}

\section{A. MTF Performance}

We have performed MTF measurements by using the spot scan method: a blackbody illuminates a pinhole placed at the focal point of a collimator, which generates a plane wave at the exit of the collimator. The camera is slightly moved in front of the collimator in order to obtain a well-sampled point spread function (PSF). Then the MTF is calculated as the Fourier transform of the PSF. Figure 9 shows the MTF of all the channels of the camera for different values of the FOV, compared to the theoretical MTF data provided by the optical design software Zemax after taking into account the transfer function of the pixel. The spatial frequency axis has a maximum value of

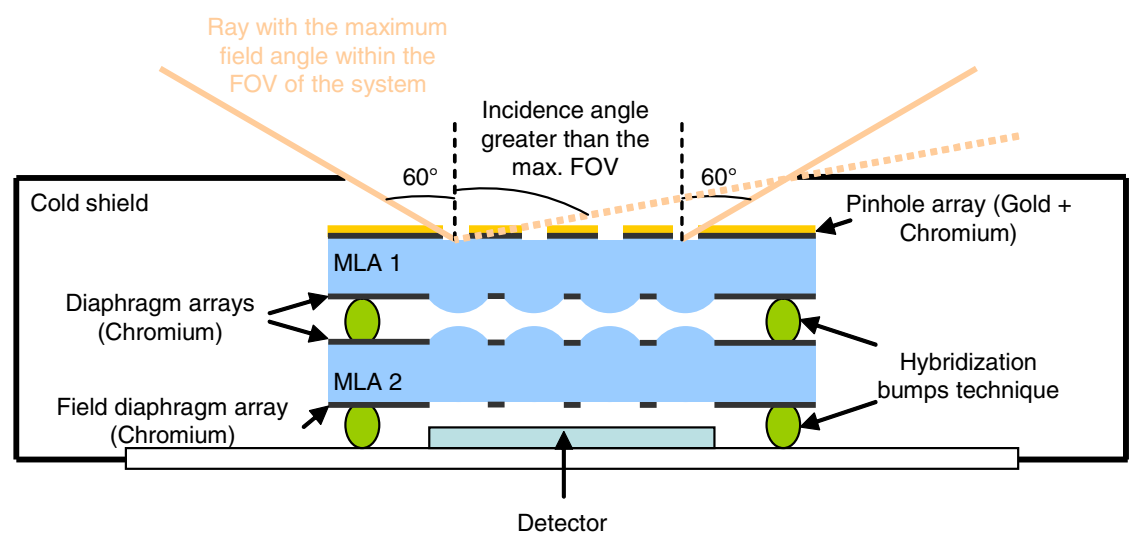

Fig. 7. (Color online) Schematic layout of the overall system exhibiting the arrays of diaphragms that prevent crosstalk between several channels (MLA: microlens array). 


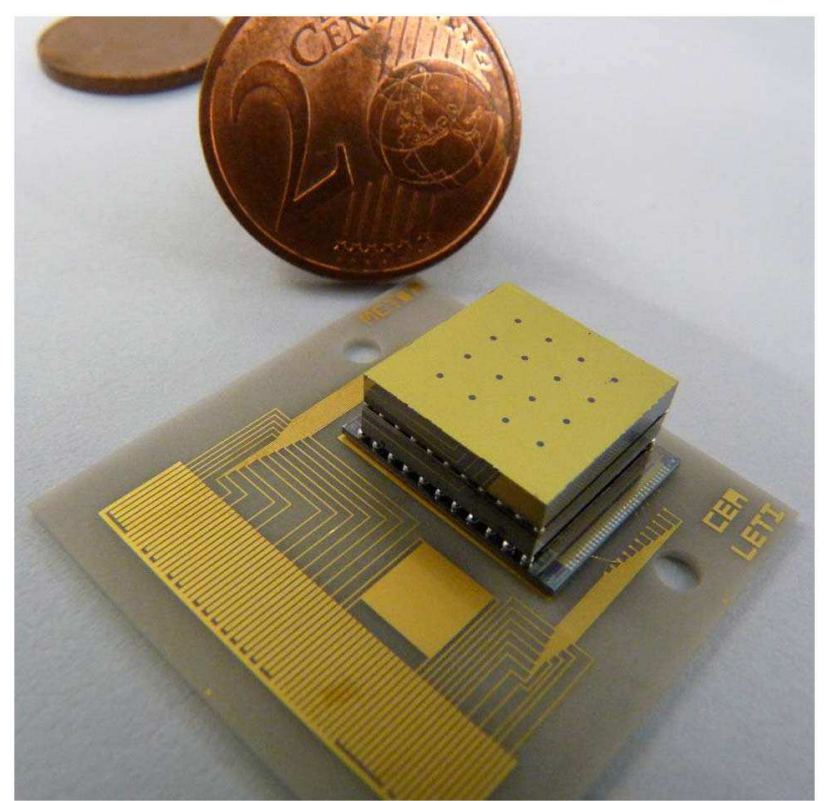

Fig. 8. (Color online) Photograph of the infrared wafer-level camera (compared to the size of a two-cent Euro coin).

67 Cycles/mm, which corresponds to the Nyquist frequency associated with an FPA of sampling pitch of $7.5 \mu \mathrm{m}$. All the channels have similar MTF curves, which means that they are identical from an optical point of view (which is a fundamental hypothesis for image processing, as explained in the next subsection). The quality of the optical system is almost limited by the diffraction, as expected from the theoretical study.

\section{B. Image Reconstruction}

We have developed an algorithm based on the classic shift-and-add technique [21] to reconstruct a wellsampled image from the set of undersampled subimages acquired by the camera. It is composed of two main steps: the registration step consists in determining the relative shifts between the subimages with subpixel accuracy, and the reconstruction step aims at adding all the subimages for which the shifts have been corrected using the registration parameters.

First, the subimages are artificially upsampled by a factor $C$ (theoretically, for our system, $C=4$ ) in order to provide an artificial finer sampling grid for the future reconstructed image.

Then, the registration step relies on the twodimensional correlation between one of the upsampled subimages (which is chosen as a reference) and the other ones. The phase correlation [29] exhibits a two-dimensional thin spot. The coordinates of this correlation peak have to be determined with

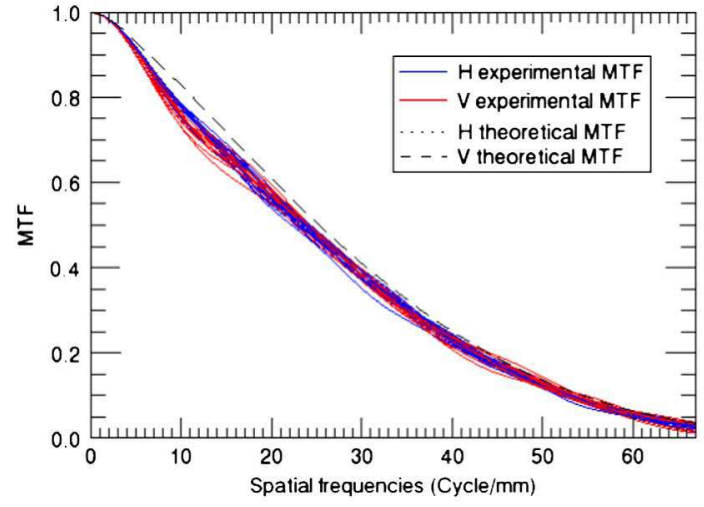

(a)

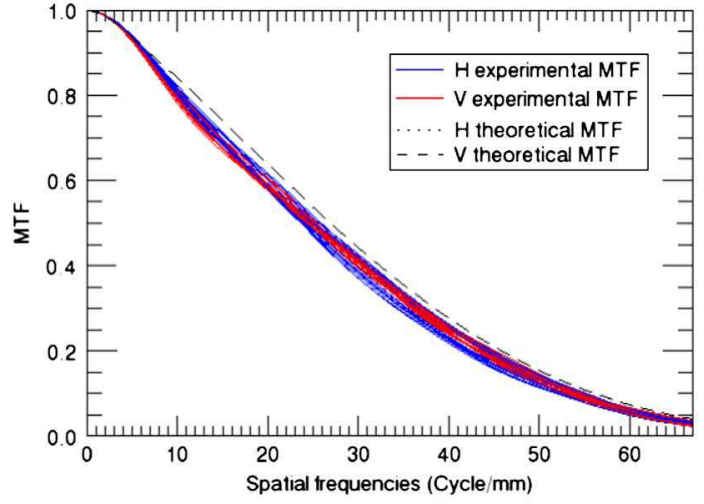

(b)

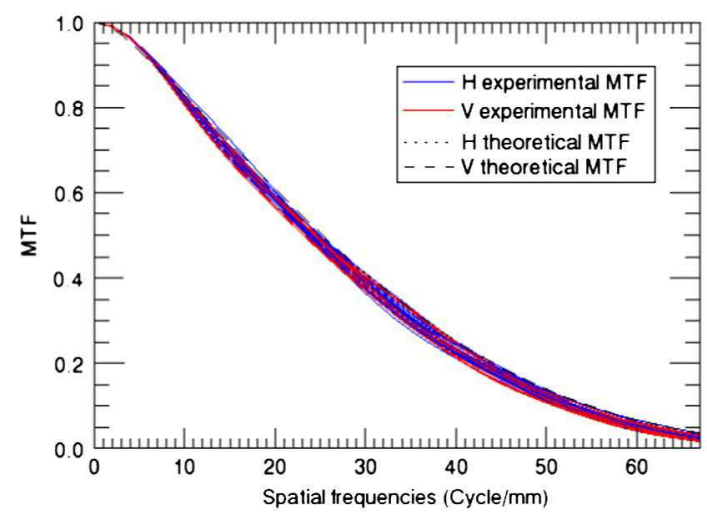

(c)

Fig. 9. (Color online) MTF measurements for all the channels of the camera and for different values of the FOV. (a) FOV $=0^{\circ}$, (b) FOV $=37^{\circ}$, and (c) FOV $=50^{\circ}$. The experimental curves are compared to the theoretical data obtained with the software Zemax. The abbreviations $\mathrm{H}$ and $\mathrm{V}$ stand for horizontal and vertical, respectively. 
subpixel accuracy, which is done by calculating the center-of-mass of the correlation peak. It is worth mentioning that, due to parallax, the relative shifts between the subimages depend on object distance $D$ : a calibration step can be carried out by acquiring various scenes in a laboratory setting at several distances of the camera. When a set of parameters is determined in this way, it can be saved as calibration data because it is constant for scenes of fixed depth.

Finally, the reconstruction step consists in adding the upsampled subimages for which the relative shifts are corrected thanks to the registration parameters. In this way, subpixel information becomes available, leading to the retrieval of high spatial frequencies [24].

We imaged a scene in a laboratory setting; the distance between the camera and the scene is equal to $D=1.60 \mathrm{~m}$. The scene is composed of a target (illuminated with a flat blackbody at the temperature of $305 \mathrm{~K})$ and a person in the same plane. The raw image acquired by the camera is shown in Fig. 10(a). Figures 10(b) and 10(c) show one of the undersampled subimages and a linear interpolation of this subimage, respectively. The result of the shift-andadd algorithm is given in Fig. 10(d): the improvement in resolution is clearly visible with respect to the linear interpolation of one subimage (for instance, the period and orientation of the slits of the target are not retrieved with the interpolation method, whereas they are with the shift-and-add algorithm).

Then, we imaged comparable scenes at various distances from the camera to determine the set of registration parameters for such fixed depths. For each value of the distance $D$ between the scene and the camera, we compare one of the subimages with the image obtained by applying the shift-and-add technique (cf. Fig. 11). For each distance, we can see that image postprocessing really improves the resolution of one subimage.

\section{Noise Equivalent Temperature Difference (NETD)}

Studying noise in multiaperture systems is an important issue that has already been addressed in the literature $[15,30-32]$. We propose here to evaluate the NETD of the cryogenic wafer-level camera. NETD is a figure of merit that is often used to characterize the temperature resolution of an infrared system: it is defined as the temperature difference for which the signal-to-noise ratio (SNR) is unitary. To measure the NETD, we placed a flat blackbody in front of the cryogenic multichannel camera. We

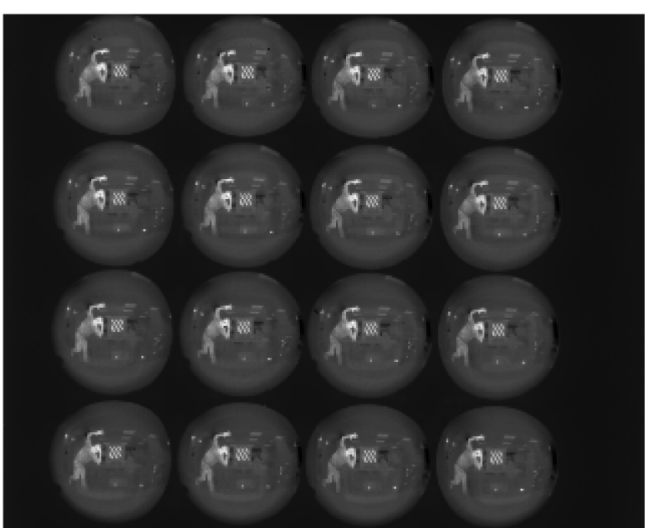

(a)

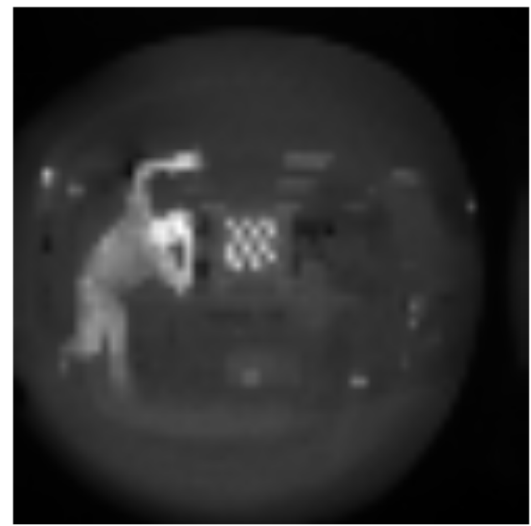

(c)

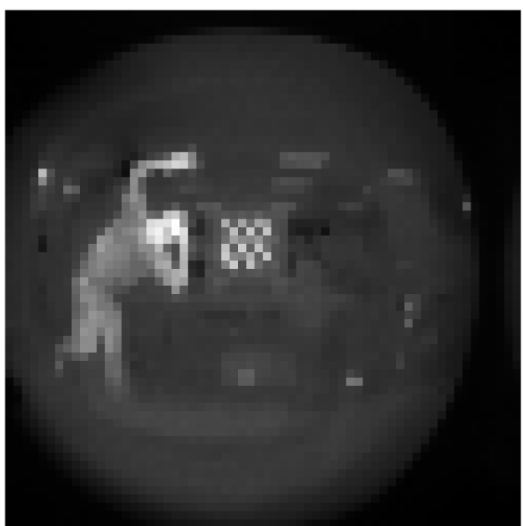

(b)

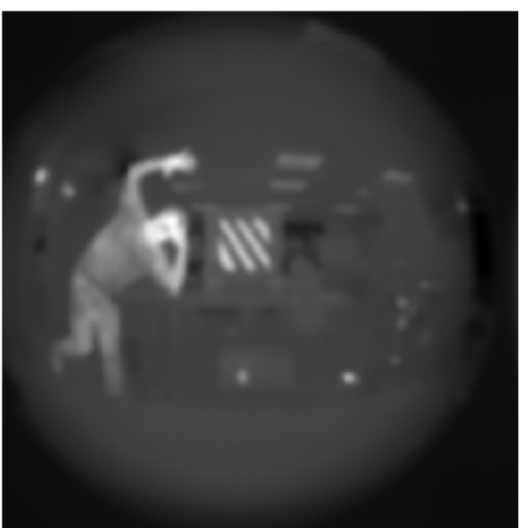

(d)

Fig. 10. Image of a scene in a laboratory setting for a distance between the camera and the scene equal to $1.60 \mathrm{~m}$. (a) Raw image acquired by the multichannel camera. (b) One of the undersampled subimages. (c) Linear interpolation of the undersampled subimage (b). (d) Image obtained with the shift-and-add algorithm. 


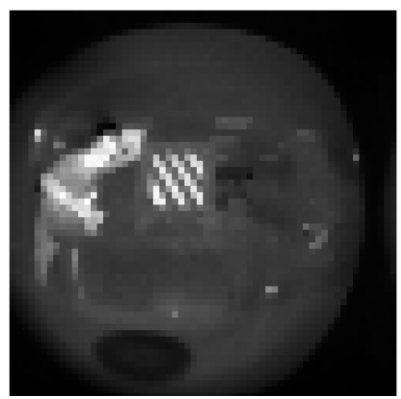

(a)

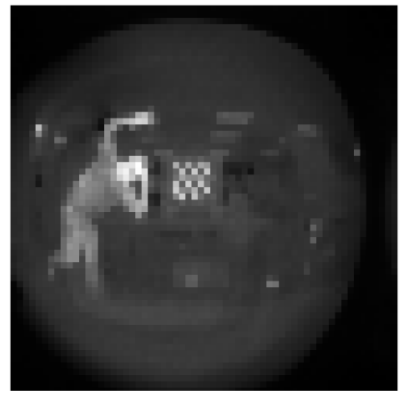

(c)

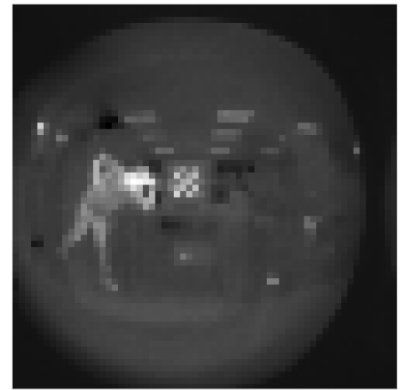

(e)

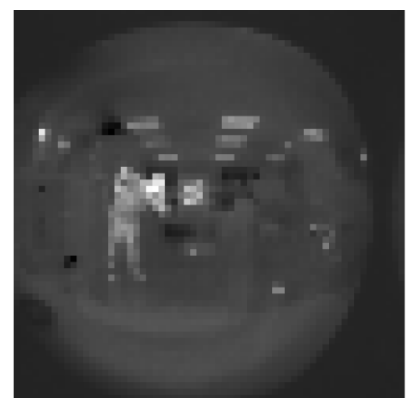

(g)

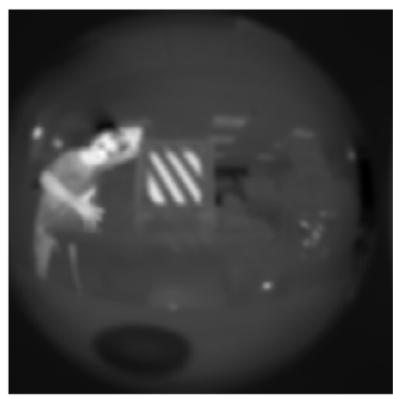

(b)

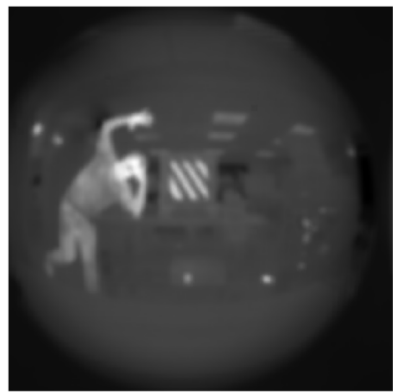

(d)

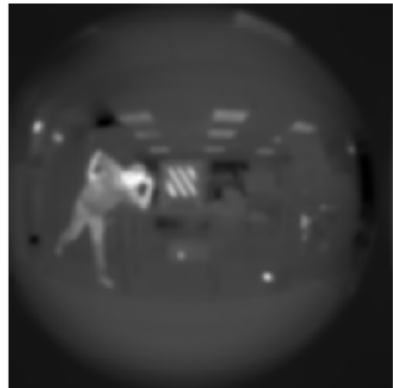

(f)

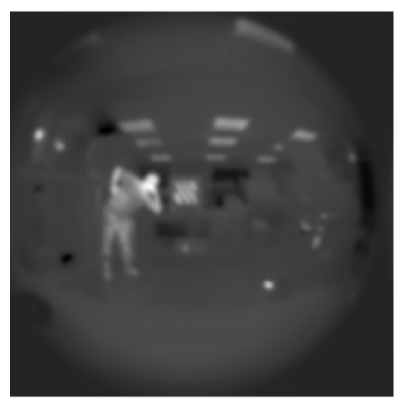

(h)
Fig. 11. Subimages acquired at distance (a) $D=1.20 \mathrm{~m}$, (c) $D=1.60 \mathrm{~m}$, (e) $D=2.20 \mathrm{~m}$, (g) $D=2.94 \mathrm{~m}$. Image obtained with the shift-and-add algorithm at distance (b) $D=1.20 \mathrm{~m}$, (d) $D=1.60 \mathrm{~m}$, (f) $D=2.20 \mathrm{~m}$, (h) $D=2.94 \mathrm{~m}$.

changed the temperature of this blackbody, from an ambient value $T_{A}$ to a hot value $T_{H}$. NETD was calculated from these measurements by using the following equation:

$$
\mathrm{NETD}=\frac{T_{H}-T_{A}}{\mathrm{SNR}}=\frac{T_{H}-T_{A}}{\frac{\operatorname{mean}\left(\operatorname{Img}_{T_{H}}\right)-\operatorname{mean}\left(\operatorname{Img}_{T_{A}}\right)}{\operatorname{std}\left(\operatorname{Img}_{T_{H}}\right)}},
$$

where $\operatorname{Img}_{T_{H}}$ and $\operatorname{Img}_{T_{A}}$ are the arrays of pixel values corresponding to the images acquired at temperatures $T_{H}$ and $T_{A}$, respectively. The signal is defined as the difference between the mean values of these two arrays. The noise is determined by calculating the standard deviation of the array of pixels acquired at temperature $T_{H}: \operatorname{std}\left(\operatorname{Img}_{T_{H}}\right)$. The difference between the two values $T_{A}$ and $T_{H}$ must not be too high to stay in the linear regime of validity of Eq. (9). From a practical point of view, we chose $T_{A}=$ $293 \overline{\mathrm{K}}$ and $T_{H}=298 \mathrm{~K}$. Then, the NETD is measured around a temperature equal to $295 \mathrm{~K}$. For a single optical axis, NETD $_{T=295 \mathrm{~K}}^{\text {one channel }}=15 \mathrm{mK}$ for $50 \%$ well fill. It is worth mentioning that we obtained a NETD equal to $20 \mathrm{mK}$ with a camera made of a single traditional lens integrated in the Dewar (described in [22]).

As a comparison, the system of [15] operates in the LWIR spectral range with an uncooled microbolometer array and has an NETD equal to $131 \mathrm{mK}$. Thus, the gain in temperature resolution is close to 10 thanks to the use of a cooled detector and of a cooled optics.

\section{Conclusion}

We have designed, manufactured, and characterized a thin cryogenic multichannel wafer-level infrared camera, working in the $3-5 \mu \mathrm{m}$ spectral range and directly integrated on a cooled IRFPA. This prototype is based on the TOMBO principle; each channel has the same wide FOV equal to $120^{\circ}$ and a very short focal length (the total track length of the camera is equal to $4.08 \mathrm{~mm}$ ). It is efficiently prevented from crosstalk between the channels thanks to the use of the cold shield and of a field diaphragm array. The optical system could be fabricated by stateof-the-art microoptics technologies, and it has been integrated on the IRFPA using original and precise hybridization techniques; that is why the camera is compatible with both cryogenic constraints and wafer-level fabrication. The resolution of a single subimage can be highly improved by applying a simple shift-and-add postprocessing algorithm to the set of undersampled raw subimages acquired by the camera. By cooling the whole system at liquid nitrogen temperature, the NETD is significantly reduced with respect to traditional infrared cameras. Within future work, we are going to study the impact of the shift-and-add postprocessing algorithm on the NETD of the reconstructed image.

\section{Appendix A: Expressions of $F$ and BFL for a Thick Bi-Convex Lens Using Ray-Transfer Matrix Formalism}

In this appendix, we give the detailed calculation of the focal length $f$ and of the BFL for a bi-convex lens with radii of curvature $R_{1}$ and $R_{2}\left(R_{1}>0\right.$ and $\left.R_{2}<0\right)$ (cf. Fig. 4).

The refractive index of the lens material is $n$. Refraction at the interface of radius $R_{1}$ is described by the following transfer matrix: 


$$
M_{1}=\left(\begin{array}{cc}
1 & 0 \\
\frac{1-n}{R_{1}} & 1
\end{array}\right)
$$

Propagation through free space of index $n$ results in the following transfer matrix:

$$
M_{2}=\left(\begin{array}{cc}
1 & \frac{d}{n} \\
0 & 1
\end{array}\right)
$$

The refraction on the interface of radius $R_{2}$ is modeled as

$$
M_{3}=\left(\begin{array}{cc}
1 & 0 \\
\frac{n-1}{R_{2}} & 1
\end{array}\right)
$$

Then, the transfer matrix $M_{\text {lens }}$ of the thick lens is calculated by $M_{\text {lens }}=M_{3} M_{2} M_{1}$, so that

$$
M_{\mathrm{lens}}=\left(\begin{array}{cc}
1+\frac{d}{R_{1}} \frac{1-n}{n} & \frac{d}{n} \\
\frac{n-1}{R_{2}}\left(1+\frac{d}{R_{1}} \frac{1-n}{n}\right)+\frac{1-n}{R_{1}} & \frac{n-1}{R_{2}} \frac{d}{n}+1
\end{array}\right) .
$$

The propagation in free space on the distance BFL is modeled by the following matrix:

$$
M_{4}=\left(\begin{array}{cc}
1 & \mathrm{BFL} \\
0 & 1
\end{array}\right)
$$

The definition of the focal length $f$ implies that $\alpha_{\text {out }}=-\frac{h_{\text {in }}}{f}$ (the angles are oriented according to anticlockwise direction), where $h_{\text {in }}$ is the height of the incident ray. Then, Eq. ( $\underline{8})$ can be written as

$$
\left(\begin{array}{c}
0 \\
-\frac{h_{\text {in }}}{f}
\end{array}\right)=\left(\begin{array}{cc}
1 & \text { BFL } \\
0 & 1
\end{array}\right) M_{\text {lens }}\left(\begin{array}{c}
h_{\text {in }} \\
0
\end{array}\right) .
$$

By solving this equation, we find

$$
\frac{1}{f}=(n-1)\left(\frac{1}{R_{1}}-\frac{1}{R_{2}}\right)+\frac{(n-1)^{2}}{n} \frac{d}{R_{1} R_{2}},
$$

and

$$
\mathrm{BFL}=f\left(1-\frac{d}{R_{1}} \frac{n-1}{n}\right)
$$

This project was sponsored by the DGA, the French procurement agency. The authors would like to thank SUSS MicroOptics for the production of high-quality microlens arrays with an important sag height, which correspond to current state-ofthe-art microoptics technology.

\section{References}

1. A. W. Lohmann, "Scaling laws for lens systems," Appl. Opt. 28, 4996-4998 (1989).

2. R. Völkel, M. Eisner, and K. J. Weible, "Miniaturized imaging systems," Microelectron. Eng. 67-68, 461-472 (2003).

3. J. Tanida, T. Kumagai, K. Yamada, S. Miyatake, K. Ishida, T. Morimoto, N. Kondou, D. Miyazaki, and Y. Ichioka, "Thin observation module by bound optics (TOMBO): concept and experimental verification," Appl. Opt. 40, 1806-1813 (2001).

4. J. Duparré, P. Dannberg, P. Schreiber, A. Bräuer, and A. Tünnermann, "Artificial apposition compound eye fabricated by micro-optics technology," Appl. Opt. 43, 4303-4310 (2004).

5. J. Duparré, P. Schreiber, A. Matthes, E. Pshenay-Severin, A. Bräuer, and A. Tünnermann, "Microoptical telescope compound eye," Opt. Express 13, 889-903 (2005).

6. J. Duparré, P. Dannberg, P. Schreiber, A. Bräuer, and A. Tünnermann, "Thin compound-eye camera," Appl. Opt. 44, 2949-2956 (2005).

7. K. Stollberg, A. Brückner, J. Duparré, P. Dannberg, A. Bräuer, and A. Tünnermann, "The Gabor superlens as an alternative wafer-level camera approach inspired by superposition compound eyes of nocturnal insects," Opt. Express 17, 15747-15759 (2009).

8. G. Druart, N. Guérineau, R. Haïdar, S. Thétas, J. Taboury, S. Rommeluére, J. Primot, and M. Fendler, "Demonstration of an infrared microcamera inspired by Xenos Peckii vision," Appl. Opt. 48, 3368-3374 (2009).

9. A. Brückner, J. Duparré, F. Wippermann, R. Leitel, P. Dannberg, and A. Bräuer, "Ultra-compact close-up microoptical imaging system," Proc. SPIE 7786, 77860A-177860A-8 (2010)

10. A. Brückner, J. Duparré, R. Leitel, P. Dannberg, A. Bräuer, and A. Tünnermann, "Thin wafer-level camera lenses inspired by insect compound eyes," Opt. Express 18, 24379-24394 (2010).

11. J. Meyer, A. Brückner, R. Leitel, P. Dannberg, A. Bräuer, and A. Tünnermann, "Optical cluster eye fabricated on waferlevel," Opt. Express 19, 17506-17519 (2011).

12. L. C. Laycock and V. A. Handerek, "Multi-aperture imaging device for airborne platforms," Proc. SPIE 6737, 6737091-673709-11 (2007).

13. L. C. Laycock and V. A. Handerek, "Miniature imaging devices for airborne platforms," Proc. SPIE 7113, 71130M1-71130M-9 (2008).

14. M. Shankar, R. Willett, N. Pitsianis, T. Schulz, R. Gibbons, R. Te Kolste, J. Carriere, C. Chen, D. Prather, and D. Brady, "Thin infrared imaging systems through multichannel sampling," Appl. Opt. 47, B1-B10 (2008).

15. A. Portnoy, N. Pitsianis, X. Sun, D. Brady, R. Gibbons, A. Silver, R. Te Kolste, C. Chen, T. Dillon, and D. Prather, "Design and characterization of thin multiple aperture infrared cameras," Appl. Opt. 48, 2115-2126 (2009).

16. A. Papoulis, "Generalized sampling expansion," IEEE Trans. Circuit Syst. 24, 652-654 (1977).

17. Y. Kitamura, R. Shogenji, K. Yamada, S. Miyatake, M. Miyamoto, T. Morimoto, Y. Masaki, N. Kondou, D. Miyazaki, J. Tanida, and Y. Ichioka, "Reconstruction of a high-resolution image on a compound-eye image-capturing system," Appl. Opt. 43, 1719-1727 (2004).

18. K. Nitta, R. Shogenji, S. Miyatake, and J. Tanida, "Image reconstruction for thin observation module by bound optics by using the iterative backprojection method," Appl. Opt. 45, 2893-2900 (2006).

19. K. Choi and T. J. Schulz, "Signal-processing approaches for image-resolution restoration for TOMBO imagery," Appl. Opt. 47, B104-B116 (2008).

20. A. V. Kanaev, J. R. Ackerman, E. F. Fleet, and D. A. Scribner, "TOMBO sensor with scene-independent superresolution processing," Opt. Lett. 32, 2855-2857 (2007).

21. J. Shi, S. E. Reichenbach, and J. D. Howe, "Small-kernel superresolution methods for microscanning imaging systems," Appl. Opt. 45, 1203-1214 (2006).

22. G. Druart, F. de la Barrière, N. Guérineau, J. Deschamps, M. Fendler, N. Lhermet, J. Rullière, S. Magli, Y. Reibel, and J.-B. 
Moullec, "Towards infrared DDCA with an imaging function," Proc. SPIE 8012, 801228-1-801228-11 (2011).

23. F. de la Barrière, G. Druart, N. Guérineau, and J. Taboury, "Design strategies to simplify and miniaturize imaging systems," Appl. Opt. 50, 943-951 (2011).

24. F. de la Barrière, G. Druart, N. Guérineau, J. Taboury, J. Primot, and J. Deschamps, "Modulation transfer function measurement of a multichannel optical system," Appl. Opt. 49, 2879-2890 (2010).

25. R. Gläbe and O. Riemer, "Diamond machining of micro-optical components and structures," Proc. SPIE 7716, 7716021-771602-10 (2010).

26. http://www.suss-microoptics.com.
27. J. W. Goodman, Introduction to Fourier Optics, 3rd ed. (Roberts and Company, 2005), p. 444.

28. M. J. Kidger, Fundamental Optical Design (SPIE, 2002).

29. P. Refregier, "Optimal trade-off filters for noise robustness, sharpness of the correlation peak, and Horner efficiency," Opt. Lett. 16, 829-831 (1991).

30. M. W. Haney, "Performance scaling in flat imagers," Appl. Opt. 45, 2901-2910 (2006).

31. M. W. Haney, "Comments on "Design and characterization of thin multiple aperture infrared cameras," Appl. Opt. 50, 1584-1586 (2011).

32. D. J. Brady, "Reply to "Comments on multiple aperture cameras," Appl. Opt. 50, 1587-1592 (2011). 